\title{
The new era in the treatment of deep vein occlusion
}

\begin{abstract}
A non-invasive, conservative treatment has been a standard in treating acute and chronic deep vein thrombosis. This treatment turned out to be ineffective, particularly in the hip area. Also, it was demonstrated that it does not influence the frequency of manifestations of post-thrombotic syndrome. Previous attempts to surgically reconstruct deep veins, unlike arteries reconstruction, yielded no positive results and also increased hemorrhagic and embolic complications. Currently, already in the period of the acute thrombosis of deep veins, the methods of early re-canalization, both with the application of targeted thrombolisis, as well as of pharmacomechanical methods, are applied.

Thanks to a wide array of image examination methods applied in pre-operational and intra-operational diagnostics optimum, it is possible to plan a revascularising treatment in the sick individuals suffering from the already developed manifestations of the post-thrombotic syndrome.

The development of endovascular methods, made possible thanks both to the surgeons' experience in the re-canalization field, as well as constant improvements of stents dedicated to the venous system, allowed for effective use of these techniques in curing the occlusion of deep veins. It was the case with the arterial system and works here as well. Applying the hybrid proceeding, combining opened techniques and endovascular ones, works very well in selected cases.
\end{abstract}

Keywords: post-thrombotic syndrome, DVT-deep venous thrombosis, venous stenting.

DOI: $10.1515 /$ pjph-2015-0020

\section{INTRODUCTION}

It is estimated that one person out of a thousand living in the West European countries, experiences deep vein thrombosis syndrome (DVT) every year. In about $40 \%$ of these cases, thrombosis develops in the hip-thigh region to a considerable degree disadvantaging outflow of blood from the limb [1].

A non-invasive treatment used to be a standard in DVT treatment. It is an ineffective treatment method, particularly in the hip-thigh area, since the re-canalization of the obstructed section is effective only in $15-30 \%$ of cases. In other cases, venous hypertensison occurs, inducing exaggerated distension of veins located below the occlusion. Other consequences include a valvular failure, as well as other postthrombotic syndrome effects, like venous limping or chronic ulcerations [2].

In the past, there were numerous attempts to perform surgical thrombectomies and systemic thrombolisis. However, the effectiveness of such therapy methods was insufficient and there were numerous complications, too. Furthermore, in case of strong manifestations of the postthrombotic syndrome, there were attempts of surgical recanalization.

Unlike it is the case with similar treatments in the arterial system, these actions were often ineffective, which led to their discontinuation in numerous facilities. At the moment, with endovascular methods having been applied for many years, special stents dedicated to the venous systems are being developed. They have different properties than those applied for arteries. Similarly, the principles of conducting treatments in the system of deep veins differ from principles applied at reconstructions of arteries, and small nuances often determine the effectiveness of the treatment. The majority of vein re-canalization attempts is done through the use of the endovascular method. In selected cases, the hybrid method, supplemented with surgeon's reconstruction, is implemented.

\section{AIM}

The purpose of the study was to present modern, endovascular procedures suitable for treating sharp thrombosis and chronic occlusion of deep veins.

\section{DISCUSSION}

\section{Qualification for the procedure}

The ultrasound scan remains to be the basic imaging examination. Right now, it is possible to examine the level of iliac veins and the inferior vena cava separately from veins of the lower limbs. Unfortunately, in case of most patients 
undergoing an ultrasound Doppler, doctors pay attention to the area below the crural arch, almost entirely ignoring the hip area. Using an ultrasound scan to assess iliac veins is difficult, because of their deep location. It needs emphasizing that only on the condition that an experienced person performs the examination, some authoritative results can be produced.

The following examination is CT venography and MR venography, along with spatial reconstructions of obtained images. They allow for showing: the compression of iliac veins (as in May-Turner syndrome), of post-thrombotic changes, such as serial occlusions and narrowings or trabeculations in the lumen of veins as well the developed collateral circulation. Using this method hugely improves the visualization of the inferior vena cava, at least when compared to an ultrasound scan. Substantial occlusion can be recognized, if performed examinations demonstrate the reduction of the lumen of the of the flow bigger than the $50 \%$ with simultaneous exposure of collaterals to lateral circulation [3].

One should plan to gain access to the further, invasive diagnostics or the reconstruction treatment after an accurate analysis of the blood inflow quality assessed through noninvasive examinations, as mentioned above.

The evaluation of the patency of the popliteal vein, thigh vein, the inflow from the deep vein of the thigh is crucial. Invasive examinations made for diagnostic purposes and as the element of the treatment of endovascular reconstruction, are phlebography and endovascular ultrasonography (IVUS). Phlebography is a great way of displaying occlusions, as well as the collateral circulation, however, as two-dimensional examining can hardly match IVUS examining during the evaluation of compression to the vein and during the assessment of the degree of narrowing. Additionally, IVUS is showing much better intravenous remains of thromboses as trabeculations, which can be hidden by the contrast during phlebography [4]. IVUS also allows to assess the wall of the vein with its possible thickening and the hyperplasia of the inside film.

\section{Intraoperative examination}

Despite the precise, preoperative evaluation in CT and MR-phlebography, on the basis of which the treatment is planned, the conclusive assessment of venous pathology is being verified in the course of the treatment. The traditional, two-dimensional phlebography would not always enable a precise evaluation of the flow channel. Phlebography carried out in a-p and oblique projection allows finding the appropriate channel for re-canalization. Possibilities of stereotactic depicting with $3 \mathrm{D}$ (like-CT) reconstruction are becoming helpful. The image is being created, thanks to the fact that the lamp in the X-ray generator revolves around the patient, during the phlebography. This visualization is peculiarly helpful in case of compression from the outside and in case of bending of the stent [5].

IVUS, used as the element of an intraoperative diagnostics, allows for a precise implantation of the stent's place.

\section{Recanalization of veins}

Acute venous thrombosis

\section{Thrombolysis}

Systemic thrombolysis enables faster recanalization of the thrombosed veins. This reduces the risk of the post-thrombotic syndrome. Despite that, the risk of some hemorrhagic complications remains high [6]. An effective recanalization, burdened with a smaller risk, is possible through using the methods of application of targeted thrombolisis and of pharmacomechanical methods. Targeted thrombolisis is done through using special, multiperforated catheters, enabling the passage of a thrombolytic directly to the thrombus. At the moment, the recommended medicine is alteplasa - recombined, endogenic plasminogen activator (Actilyse ${ }^{\circledR}$, Boehringer-Ingelheim, Germany), applied in the dose of $20 \mathrm{mg} / 24$ hours, at the maximum recommended intake time of 96 hours [1]. In the time of thrombolisis, one should keep the intake of heparin at a stable level, extending it 1.2-1.7-fold of kaolin-kefalin time (APTT) [7].

After thrombolisis is performed, its effectiveness is being assessed, through the use of phlebography and IVUS. As much as $80 \%$ of the sick individuals with the hip-thigh thrombosis, are diagnosed with May-Thurner syndrome. It is associated with the pressure in the right common iliac artery on the left common iliac vein. CT examinations show that $31 \%$ of the population reports compression above $70 \%$ [8]. However, only in some individuals, compression causes the appearance of clinical symptoms such as the deep vein thrombosis, limb pain or in the pelvis, swelling of the limb.

Women are more likely to suffer from May-Thurner syndrome which is one of the most undermined causes of deep vein thrombosis syndrome in pregnant women [9].

The angioplasty, using the stent, is essential for determining the compression of iliac veins [10], because the lack of mechanical propping of the compressed vein pressed down exposes patients to the recurrence of deep vein thrombosis syndrome, as well as increasing the risk of the development of the post-thrombotic syndrome [11]. It is necessary to put a stent in all areas, in which residual narrowing is visible after trombolisis.

\section{Pharmacomechanical methods}

In pharmacomechanical methods, the thrombus is being mechanically grinded down, which is supposed to increase the surface of the thrombolitic action. This shortens the procedure [12]. The following systems are applied:

- Trellis: Venous section subjected to the treatment is limited by 2 balloons, between which there is a quickly rotating wire (3500 revolutions per minute).

- AngioJet: Thrombus is broken by strong rivulets of water, next sucked out to the catheter. The application of the system allows recanalization during one treatment.

- Ekos system: A wire containing micro converters producing the high-frequency ultrasonic waves is placed inside the thrombus. They are supposed to improve the penetration of the thrombolitic agent given by the system at the same time.

\section{Chronic obstruction of illiac veins}

Endovascular recanalization treatment of changed postimflammatory deep veins, is conducted under general anesthesia. Balloon angioplasty has proven ineffective in the attempts of the venous system recanalization [3]. This refers to the elastic, post-thrombotic changes, which ensures the long-term patency of an appropriately implanted stent. Dedicated stents are applied in the venous system and they are 
characterized by a great radial power, particularly necessary in compression syndromes. The stent in the venous system should also be characterized by great flexibility, which could be provided by self-compressing stents. The stent decompressed on the balloon is very susceptible to breakdowns, particularly in the hip area - in the place of joining the external iliac vein and internal one.

Within the last few years, many stents dedicated to be used in the venous system have come into existence. Stents are designed with a woven structure, they closed and opened cellular, character as well as hybrid stents, combining both features. Venous stents differ in parameters: radial power and the flexibility, available diameters and lengths. "These differences are being taken advantage of at the choice of the appropriate stent for the cleared region of the vein e.g. other stent to post-inflammatory obstructed inferior vena cava, other to the common iliac vein pressed down by the artery in the May-Turner syndrome". Currently, the researchers are still doubtful about the effectiveness of individual types of stents applied in specific regions of the venous system and depending on the cause of occlusions. There is still a huge demand for the polycentric clinical research, looking at the long-term effectiveness of individual stent types [13].

Recanalization of the hip area can be ineffective, even if a stent of high radial strength is used, particularly in case of the insufficient inflow to the stented segment. Physiologically, the inflow to the level of the common femoral vein is ensured by the femoral vein, the deep vein of the thigh and the saphenous vein. A deep vein of the thigh is a crucial source of the influx. In most cases, provided that the veins remain passable, even post-thrombotic occlusion of the femoral vein will not stop the inflow to the stented hip segment. However, given the presence of organized post-inflammatory changes in the place of the flow of the deep vein, the permanence of the endovascular treatment is limited. Perhaps then, the hybrid method may prove effective, combining stenting of the hip segment with surgical endophlebectomy of the common hip vein and deep femoral vein. Endophlebectomy should, then, be supplemented with plastic, using a patch and the temporary arteriovenous fistula, ensuring an increased flow during the first week of reconstruction [14].

\section{CONCLUSIONS}

An early removal of the thrombus, with the help of targeted thrombolisis and of pharmaco-mechanical methods, lowers the risk of post-thrombotic syndrome.

Thanks to the current endovascular techniques, reducing the manifestations of post-thrombotic syndrome is easier, thanks to the treatment methods of the venous hypertension generated by occlusions of veins in the hip section.

\section{REFERENCES}

1. Wittens C. Best practice in venous procedures. Italy: Edizioni Minerva Medica; 2010.

2. Saarinen J, Kallio T, Lehto M, et al. The occurrence of the post-thrombotic changes after an acute deep vein thrombosis. A prospective twoyear follow-up study. J Cardiovasc Surg (Torino). 2000;41(3):441-6.

3. Neglen P, Raju S. Proximal lower extremity chronic venous outflow obstruction: recognition and treatment. Semin Vasc Surg. 2002;15:5764.

4. Neglen P, Raju S. Intravascular ultrasound scan evaluation of the obstructed vein. J Vasc Surg. 2002;35:694-700.

5. Hsieh MC, Chang PY, Hsu WH, et al. Role of three-dimensional rotational venography in evaluation of the left iliac vein in patients with chronic lower limb edema. Int J Cardiovasc Imaging. 2011;27:9239.

6. Watson LI, Armon MP. Thrombolysis for acute deep vein thrombosis. Cochrane Database Syst Rev. 2004;4:CD002783.

7. Ly B, Njaastad AM, Sandbaek G, et al. Catheter-directed thrombolysis of iliofemoral venous thrombosis. Tidsskr Nor Laegeforen. 2004;124:47880.

8. Nazzal M, El-Fedaly M, Kazan V, et al. Incidence and clinical significance of iliac vein compression. Vascular. 2014 Nov 14. pii: 1708538114551194 . [Epub ahead of print]

9. Wax JR, Pinette MG, Rausch D, Cartin A. May-Thurner syndrome complicating pregnancy: a report of four cases. J Reprod Med. 2014;59(56):333-6.

10. Chung JW, Yoon CJ, Jung SI, et al. Acute iliofemoral deep venous thrombosis: evaluation of underlying anatomic abnormalities by spiral venography. J Vasc Interv Radiol. 2004;15:249-56.

11. Mathur M, Cohen M, Bashir R. May-Thurner syndrome. Circulation. 2014;129(7):824-5.

12. Rao AS, Konig G, Leers SA, et al. Pharmacomechanical thrombectomy for iliofemoral deep vein thrombosis: an alternative in patients with contraindications to thrombolysis. J Vasc Surg. 2009;50:1092-8.

13. de Graaf R, Wittens CH. Endovascular treatment options for chronic venous obstructions. Phlebology. 2012;27(Suppl. 1):171-7.

14. Vogel DCA, Al-Jabouri M, Assi ZI. Common femoral endovenectomy with iliocaval endoluminal recanalization improves symptoms and quality of life in patients with postthrombotic iliofemoral obstruction. J Vasc Surg. 2012;55:129-35.

\section{Corresponding author}

Jan Jakub Kęsik

Department of Vascular Surgery and Angiology, Medical University of Lublin

11 Staszica Str., 20-801 Lublin

E-mail: jan.kesik@wp.pl

In the Clinic of Vascular Surgery and Angiology, Medical University of Lublin, endovascular methods of early removing of the thrombus from the lumen of the veins, are applied with the help of targeted thrombolisis and mechanical thrombectomy. AngioJet system is applied.

The program implemented in a Clinic of Vascular Surgery and Angiology, Medical University of Lublin of treatment of acute and chronic occlusions of deep veins was possible thanks to experience of the leading European Centre of curing illnesses of deep veins in Maastricht. 